\title{
$\operatorname{micr} \varphi b i o l o g y \mapsto \longmapsto \mapsto v$ $\longmapsto "$ !...Comment ...?"
}

Microbiology Comment provides a platform for readers of Microbiology to communicate their personal observations and opinions in a more informal way than through the submission of papers.

Most of us feel, from time to time, that other authors have not acknowledged the work of our own or other groups or have omitted to interpret important aspects of their own data. Perhaps we have observations that, although not sufficient to merit a full paper, add a further dimension to one published by others. In other instances we may have a useful piece of methodology that we would like to share.

The Editors hope that readers will take full advantage of this section and use it to raise matters that hitherto have been confined to a limited audience.

Jon Saunders

Editor-in-Chief

\section{Agricultural and medical microbiology: a time for bridging gaps}

'Plants and humans do not generally share pathogens and there seems to be no term analogous to "zoonosis" for an infection acquired from a plant' (6). This quotation from a recent review by Fred Falkiner on the use of antimicrobials in plant science appears at first sight to provide a reassuring message, particularly at a time when the problem of multiresistant human pathogens has been highlighted $(10)$ and a Government report (12) has called for a ban on the routine use of antimicrobial agents in animal husbandry. However, Falkiner added the caveat that inherently multiresistant rhizosphere organisms, such as Burkholderia cepacia, can cause both human and plant infections, but are deemed 'environmental'. We thought it timely to introduce $B$. cepacia to a wider audience since, as with the issue of bovine spongiform encephalopathies, it reveals the unfortunate gap that exists between agricultural and medical microbiologists (1). This is now further highlighted by licence applications to national environmental agencies to use B. cepacia as a biological control agent.

B. cepacia was first described by Walter Burkholder in 1950 as the phytopathogen responsible for bacterial rot of onions (3). In their seminal taxonomic study published in this journal in the $1960 \mathrm{~s}$, Stanier and colleagues $(20)$ noted the extraordinary metabolic versatility of $B$. cepacia (synonym Pseudomonas multivorans, eater of everything), including its ability to degrade a wide range of organic compounds. The inherent resistance of the organism to antibiotics and ability to survive in nutritionally limited environments are exemplified by use of penicillin $\mathrm{G}$ as a sole carbon source (2) and its role as the major bacterial contaminant of space shuttle water supplies (15).

In the past two decades, the number of human infections caused by $B$. cepacia has increased markedly (9). The organism appears to have a predilection for the respiratory tract; life-threatening lung infections occur in patients requiring mechanical ventilation and in individuals with chronic granulomatous disease or cystic fibrosis (CF) (9). B. cepacia is a major pathogen in the $\mathrm{CF}$ population (9); pulmonary colonization reduces survival by $50 \%(7)$ and approximately one-third of patients succumb to 'cepacia syndrome', a rapidly fatal necrotizing pneumonia, sometimes accompanied by bacteraemia (9).

In contrast to its pathogenic role in plants and humans, B. cepacia is attracting considerable interest from the agricultural industry as a biological control agent $(9,11$, 17). B. cepacia can repress many soilborne plant pathogens and thus improve germination and crop yields. Its striking metabolic potential can also be exploited in the bioremediation of soils treated previously with toxic pesticides and herbicides. Government agencies in North America are presently examining licence applications for bulk use of B. cepacia for seed treatment and foliar spraying of crops. Interestingly, applications for use of B. cepacia in soil bioremediation, including the preparation of golf courses, come under the jurisdiction of a separate regulatory authority.

A major reason for agricultural interest in bacterial biopesticides is to provide environmentally friendly and less toxic agents. This is a laudable strategy provided that toxicity is not replaced by infections with an organism that is notoriously difficult to treat. Agricultural workers distributing bacterial biopesticides, or those harvesting crops or tilling fields, could be at particular risk. Water supplies and foodstuffs could also be contaminated, as B. cepacia can survive for long periods in water and other moist nutritionally deficient environments $(9,15)$. Regulatory agencies are faced with the difficult task of balancing the human hazards associated with toxicity of chemical biopesticides with potential infectious hazards presented by microbial-based preparations.

The microbiological evidence presented during the biopesticide debate merits careful scrutiny. Arguments used to justify the 'safety' of B. cepacia include claims that environmental and human isolates can be distinguished by phenotypic and molecular properties, and that there is no scientific evidence that human infections have been caused by direct acquisition of B. cepacia from the environment. However, there is compelling circumstantial evidence for an environmental source in several $B$. cepacia infections found in otherwise healthy humans; e.g. swamp foot, an invasive skin infection acquired by military personnel during jungle training (21) and fatal community-acquired pneumonia in children (16). Furthermore, clinical and environmental B. cepacia isolates share phytopathogenic

\section{- GUIDELINES}

Communications should be in the form of letters and should be brief and to the point. A single small Table or Figure may be included, as may a limited number of references (cited in the text by numbers, and listed in alphabetical order at the end of the letter). A short title (fewer than 50 characters) should be provided.

Approval for publication rests with the Editor-in-Chief, who reserves the right to edit letters and/or to make a brief reply. Other interested persons may also be invited to reply. The Editors of Microbiology do not necessarily agree with the views expressed in Microbiology Comment.

Contributions should be addressed to the Editor-in-Chief via the Editorial Office. 
potential (9) and lipopolysaccharide from both groups produces inflammatory activation of human neutrophils (13) and a proinflammatory cytokine response tenfold greater than Pseudomonas aeruginosa lipopolysaccharide (19). Finally, B. cepacia J2315, a CF isolate which represents the virulent and highly transmissible E/T12 lineage (9), produces a cytotoxin (14) whose molecular template is also present in the biopesticide strain AMMD (M. Hutchison, personal communication).

Integrated genotypic and phenotypic analyses have shown that $B$. cepacia isolates, cultured from clinical or environmental sites, belong to at least five distinct genomic species or genomovars, referred to collectively as the B. cepacia complex (22). Following identification of distinguishing phenotypic characteristics, the name Burkbolderia multivorans has been proposed for genomovar II (22) and Burkholderia vietnamiensis for genomovar $\mathrm{V}(8)$; the remaining three groups are referred to as genomovars I, III and IV pending differential phenotypic tests. All five groups have been cultured from CF patients and environmental samples.

The biological origin of the five genomovars within the B. cepacia complex is unclear. Physical and molecular evidence indicates that clinical and environmental isolates of the B. cepacia complex contain large genomes of extremely variable length. These are of the order of 4-8 $\mathrm{Mb}$, comprised of three to five replicons and containing multiple insertion sites; multiple chromosome rearrangements have also been reported $(4,11,18)$. To the best of our knowledge, this is a unique situation in bacterial classification and one can rightfully question the validity of overall genomic DNA-DNA hybridizations to delineate species within this extraordinary complex (22).

The initial delineation of three genomovars (I, III and IV) was based primarily on differences between whole-cell protein electrophoretic profiles and the level of whole-genome DNA-DNA hybridization (22). Subsequent analyses of several hundreds of isolates from CF patients, non-CF patients and environmental sources blurred the picture of three well-separated taxa and DNA-DNA hybridization experiments on numerous strains would be required to elucidate the species identity of many isolates within the B. cepacia complex. In contrast, $B$. multivorans and $B$. vietnamiensis are mostly readily identified.

Available taxonomic data indicate that there is no clear distinction between 'environmental' and 'clinical' species within the B. cepacia complex. The process of speciation within this complex, the origins of the differences in genome sizes, and the significance of the copious presence of insertion sequences are unknown, as is the potential influence of horizontal gene transfer on the evolutionary relationships within the $B$. cepacia complex. Compelling new evidence for a close relationship between environmental and clinical isolates comes from the use of PFGE and comparison of RFLPs within whole bacterial genomes. Not surprisingly, such analyses have demonstrated extensive genomic heterogeneity within the B. cepacia complex. Despite this heterogeneity, following cleavage with endonucleases $\mathrm{Xbal}$ and $S p e \mathrm{I}$, we observed identical restriction fragment patterns in the genomic DNA of the environmental $B$. cepacia type strain ATCC 25416 and a $B$. cepacia isolate cultured from the sputum of a patient with CF.

Clearly, the pathogenic potential of individual members of the $B$. cepacia complex requires clarification. However, unequivocal evidence for lack of virulence in any member of this closely related group is difficult to obtain. Ethical considerations prevent the use of human experiments to compare the virulence of environmental and clinical isolates. Repeated exposure of transgenic CF mice to the genomovar III isolate, J2315, showed more severe pathology, including pneumonia, in CF mice than in non-CF controls (5). Pulmonary clearance of a genomovar I rhizosphere $B$. cepacia strain was also found to be tenfold less than J2315 (J. Hughes, personal communication). Although this animal model is providing useful information on the pathophysiology of $\mathrm{CF}$, at present, it cannot provide unequivocal information on the potential virulence of biopesticide strains and other members of the B. cepacia complex.

We appreciate the research investment that agricultural microbiologists have made in B. cepacia biopesticides. We also understand the beneficial potential of such agents and the frustration that must be felt when the implementation of research is thwarted by regulatory authorities or specialists from another field of microbiology. Nevertheless, there is sufficient evidence to recommend extreme caution before granting of $B$. cepacia biopesticide licences. If approval is granted, then a careful surveillance must be made to monitor potential human hazards. In addition to risk of human infection caused by the original biopesticide strain, a longerterm concern is the effect that bulk distribution might have on the emergence and survival of mutant forms of the biopesticide and the molecular genetic evidence which points to the potential for horizontal gene transfer between this organism and other members of the B. cepacia complex present in environmental ecosystems. Whilst North American regulatory bodies face the daunting task of judging the advantages and disadvantages of individual $B$. cepacia biopesticides, it seems timely to test the views of UK and other European authorities. Preliminary inquiries raise the following questions:

Are plant pathogens like B. cepacia and other pseudomonads outside the remit of the UK Advisory Committee on Dangerous Pathogens on the grounds that in most cases they do not infect 'healthy' individuals?

Is it the case that so many microbes are potential pathogens to compromised hosts that the logistics of passing unequivocal judgements on any one biopesticide are too time-consuming?

Is B. cepacia not a serious enough plant pathogen to worry MAFF, and not pathogenic enough in human terms to concern the Health and Safety Executive?

Unless genetically modified, are B. cepacia biopesticides not officially under the remit of the British agriculture ministry's Pesticides Safety Directorate?

In conclusion, the $B$. cepacia complex presents a paradigm of emerging microbial challenges: these include use of molecular taxonomic analyses to clarify the phylogenetic relationship between environmental bacteria and human pathogens; the adaptability of prokaryotes with multiple replicons and genome sizes twice that of Escherichia coli; and the evolution and spread of virulence genes between multiresistant bacteria in environmental microcosms. We hope that important microbiological issues such as food poisoning, bovine spongiform encephalopathies, the emergence of multiresistant pathogens and now the debate on the benefits and hazards of bacterial biopesticides will highlight the breadth of modern microbiology and the importance of collaboration between microbiologists from different specialities. The precaution of wearing of a long-sleeved shirt, long pants, gloves and a respirator may minimize exposure of agricultural workers to dermal exposure and inhalation of $B$. cepacia biopesticides; however, these precautions do not eliminate the long-term risks to other humans, or provide the means to fund the expensive litigation should things go wrong! What better microbial challenge to unite agricultural and medical microbiologists than an organism that reduces an onion to a macerated pulp, protects other crops from bacterial and fungal disease, devastates the health and social life of CF patients, and not only is resistant to the most famous of antibiotics, penicillin, but can use it as a nutrient!

The authors are grateful to Dr Gerry Saddler for his helpful comments on UK biopesticide regulations and to Cathy Doherty for PFGE analyses. P. V. is indebted to the Fund for Scientific Research - Flanders (Belgium) for a position as post-doctoral research fellow. 


\section{John R. W. Govan"* and Peter Vandamme ${ }^{2}$}

'Department of Medical Microbiology, University of Edinburgh Medical School, Teviot Place, Edinburgh EH8 9AG, UK. 'Laboratorium voor Microbiologie, Universiteit Gent, KL Ledeganckstraat 35, B-9000 Gent, Belgium.

*For correspondence. Tel: +441316503164. Fax: +441316506531.

e-mail: John.R.W.Govan@ed.ac.uk

1. Anonymous (1996). Less beef, more brains. Lancet $347,915$.

2. Beckman, W. \& Lessie, T.G. (1979). Response of Pseudomonas cepacia to $\beta$-lactam antibiotics: utilization of penicillin $\mathrm{G}$ as the carbon source. J Bacteriol 140 , 1126-1128.

3. Burkholder, W.H. (1950). Sour skin, a bacterial rot of onion bulbs. Phytopathology 40, 115-117.

4. Cheng, H. \& Lessie, T. (1994). Multiple replicons constituting the genome of Pseudomonas cepacia 17616. J Bacteriol 176, 4034-4042.

5. Davidson, D.J. Dorin, J.R., McLachlan, G., Ranaldi, R., Lamb, D., Dohery, C., Govan, J. \& Porteous, D.J. (1995). Lung disease in the cystic fibrosis mouse exposed to bacterial pathogens. Nat Genet 9, 351-357.

6. Falkiner, F.R. (1998). The consequences of antibiotic use in horticulture. J Antimicrob Chemother 41, 429-431.

7. FitzSimmons, S. (1997). Cystic Fibrosis Foundation, National CF Patient Registry.

8. Gillis, M., Van, T.V., Bardin, R. \& 7 other authors (1995). Polyphasic taxonomy in the genus Burkholderia leading to an amended description of the genus and proposition of Burkholderia vietnamiensis sp. nov, for $\mathrm{N}_{2}$-fixing isolates from rice in Vietnam. Int J Syst Bacteriol 45, 274-289.

9. Govan, J.R.W., Hughes, J.E. \& Vandamme, P. (1996). Burkbolderia cepacia: medical, taxonomic and ecological issues. J Med Microbiol 45, 395-407.

10. Hawkey, P.M. (1998). Action against antibiotic resistance: no time to lose. Lancet 351, 1298-1299.

11. Holmes, A., Govan, J.R.W. \& Goldstein, R. (1998).

Could the agricultural use of Burkholderia

(Pseudomonas) cepacia pose a threat to human health? Emerg Infect Dis 4, 221-227.

12. House of Lords Select Committee on Science and

Technology. (1998). Resistance to Antibiotics and Other

Antimicrobial Agents. 7th Report Session 1997-1998, pp 1-108. London: The Stationery Office.

13. Hughes, J.E., Stewart, J., Barclay, G.R. \& Govan,

J.R.W. (1997). Priming of neutrophil respiratory burst

activity by lipopolysaccharide from Burkholderia cepacia. Infect Immunol 65, 4281-4287.

14. Hutchison, M.L., Poxton, I.R. \& Govan, J.R.W. (1998). Burkholderia cepacia produces an ion-channel hemolysin that is capable of inducing apoptosis and degranulation of mammalian phagocytes. Infect Immunol 66, 2033-2039.

15. Koenig, D.W. \& Pierson, D.L. (1997). Microbiology of the space shuttle water systems. Water Sci Technol 35 , 59-64.

16. Pujol, M. Corbella, X., Carratala, J. \& Gudiol, F. (1992). Community acquired bacteremic Pseudomonas cepacia pneumonia in an immunocompetent host. Clin Infect Dis 15, 887-888.

17. Reddy, M. (1997). Status on commercial development of Burkbolderia cepacia for biological control of fungal parhogens and growth enhancement of conifer seedlings for a global market. US Forest Service General Technica Report PNW 0(0389), 235-244.

18. Rodley, P.D., Romling, U. \& Tummler, B. (1995). A

physical map of the Burkbolderia cepacia type strain 17616. Mol Microbiol 17, 57-67.

19. Shaw, D., Poxton, I.R. \& Govan, J.R.W. (1995). Biological activity of Burkbolderia (Pseudomonas) cepacia lipopolysaccharide. FEMS Immunol Med Microbiol 11, 99-106.
20. Stanier, R.Y., Palleroni, N.J. \& Doudoroff, $M$. (1966). The aerobic pseudomonads: a taxonomic study J Gen Microbiol 43, 159-271.

21. Taplin, D., Bassett, D.C.J. \& Mertz, P.M. (1971).

Foot lesions associated with $P_{\text {seudomonas cepacia. }}$ Lancet 2, 568-571.

22. Vandamme, P., Holmes, B., Vancanneyt, M. \& 8 other authors (1997). Occurrence of multiple genomovars of Burkholderia cepacia in cystic fibrosis patients and proposal of Burkholderia multivorans sp. nov. Int J Syst Bacteriol 47, 1188-1200.

\section{The mvrA locus of Escherichia coli does not encode a ferredoxin-NADP+ reductase}

Ferredoxin-NADP ${ }^{+}$reductases (FNRs) are FAD-containing enzymes found in bacteria, plastids and mitochondria that catalyse the reversible electron transfer between $\operatorname{NADP}(\mathrm{H})$ and ferredoxin or flavodoxin (1). They are involved in a wide variety of redox metabolic pathways in different tissues and organisms, functioning as a universal class of electronic transducers between obligatory one- and two-electron carriers (1). The isoform present in Escherichia coli, encoded by the $f p r$ gene (2), is a member of the soxRS regulon, and is therefore involved in the concerted cell response towards superoxide intoxication (7).

In 1988, Morimyo (8) selected a number of mutagenized E. coli strains that were abnormally sensitive to the superoxide-propagating compound methyl viologen (MV). One of these strains was used as a host in complementation experiments to clone a gene (named $m v r A$ ) that was able to rescue the mutant cells from killing by MV (8). Further research showed that, despite some frameshift errors in the published sequence, the complementing gene was clearly related to $E$. coli FNR, raising the possibility that the mvrA locus could encode a ferredoxin-NADP ${ }^{+}$reductase $(2,7)$. Indeed, expression of a plasmid-borne plant FNR restored the wild-type phenotype, including MV tolerance, to mvrA mutant bacteria (5), while E. coli cells in which the fpr gene had been knocked-out by insertional mutagenesis became hypersensitive to this redox cycling compound (3).

However, several observations challenged the validity of the previous assignment. Bianchi et al. (2) showed that the murA and $\mathrm{fpr}$ loci were located at different positions in the E. coli chromosome. Also, the phenotypes displayed by the two mutant strains were markedly different $(3,6,8)$, although this discrepancy could be attributed to the different genetic backgrounds and mutagenesis procedures employed to generate them $(3,8)$. In order to solve this question, we undertook a reassessment of the relationship between these loci, by determining the expression and activity levels of FNR in $m v r A$

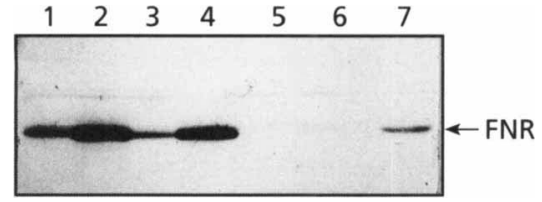

Fig. 1. Expression of ferredoxin-NADP ${ }^{+}$reductase in different $E$. coli strains. FNR immunoblots were carried out on E. coli lysates as described in the text. Cells from strains MC48 (lanes 1 and 2), murA (lanes 3 and 4) and fpr (lanes 5 and 6) were challenged (lanes 2, 4 and 6) or not (lanes 1, 3 and 5) with $0.2 \mathrm{mM} \mathrm{MV}$ prior to rupture and analysis. Lane $7,0 \cdot 2 \mu \mathrm{g}$ E. coli FNR.

and $f p r$ mutant cells under different growth regimes.

The genotypes and culture conditions of the fpr, murA and MC48 strains are described by Krapp et al. (6). To induce the soxRS response, bacterial cultures grown to the early exponential phase $\mathrm{COD}_{550}$ $\sim 0.3$ ) were incubated for $1 \mathrm{~h}$ at $37{ }^{\circ} \mathrm{C}$ in Luria-Bertani broth containing $0.2 \mathrm{mM} \mathrm{MV}$ (7). Cells were then collected, washed and ruptured by sonic oscillation (6). The presence of FNR in the various $E$. coli strains was analysed by SDS-PAGE and immunoblotting (6). Bacterial lysates were separated on $12 \%$ polyacrylamide gels, transferred to nitrocellulose membranes and immunodecorated with rabbit antisera raised against E. coli FNR (3). FNR activity in cleared lysates was determined by measuring the diaphorase reaction, namely, the ability to catalyse NADPH oxidation by artificial electron acceptors (1). In order to identify individual enzymes displaying diaphorase activity, E. coli extracts were resolved by electrophoresis in non-denaturing $10 \%$ polyacrylamide gels, and stained for activity using nitro blue tetrazolium as electron acceptor (4).

The MC48 strain, from which the mvrA mutants were derived (8), was used as an FNR-proficient control. Lysates from these bacteria show a single protein species reacting toward FNR antisera (Fig. 1, lane 1), whose levels were increased $\sim 20$-fold upon a brief challenge with MV (Fig. 1, lane 2). FNR could not be detected in the soluble fractions obtained from $\mathrm{fpr}$ cells, even after MV treatment (Fig. 1, lanes 5 and 6), as expected from a bona fide FNR-deficient strain (3). At variance, the FNR patterns observed in mvrA mutant cells were similar to those displayed by the parental strain MC48 (Fig. 1, lanes 3 and 4), indicating that the enzyme was expressed in the mutant bacteria, and readily induced in the course of the soxRS response.

These experiments cannot rule out the possibility that the FNR expressed in murA cells might be inactive. Indeed, the procedure employed to generate the mutants, random 